\title{
Automated Angiogenesis Quantification through advanced Image Processing Techniques
}

\author{
Charalampos N. Doukas, Ilias Maglogiannis, Aristotle Chatziioannou, Andreas Papapetropoulos
}

\begin{abstract}
Angiogenesis, the formation of blood vessels in tumors, is an interactive process between tumor, endothelial and stromal cells in order to create a network for oxygen and nutrients supply, necessary for tumor growth. According to this, angiogenic activity is considered a suitable method for both tumor growth or inhibition detection. The angiogenic potential is usually estimated by counting the number of blood vessels in particular sections. One of the most popular assay tissues to study the angiogenesis phenomenon is the developing chick embryo and its chorioallantoic membrane (CAM), which is a highly vascular structure lining the inner surface of the egg shell. The aim of this study was to develop and validate an automated image analysis method that would give an unbiased quantification of the micro-vessel density and growth in angiogenic CAM images. The presented method has been validated by comparing automated results to manual counts over a series of digital chick embryo photos. The results indicate the high accuracy of the tool, which has been thus extensively used for tumor growth detection at different stages of embryonic development.
\end{abstract}

\section{INTRODUCTION}

A ngiogenesis is a tightly regulated process that is essential for embryonic development that has also been implicated in physiological, as well as pathological phenomena in fully developed organisms [1]. Soluble growth factors, adhesion molecules, proteases, as well as the extracellular matrix have been shown to interact spatially and temporally to co-ordinate the formation of new blood vessels. Stimulation of new blood vessel growth has been proposed as a therapeutic approach to treat conditions that include ischemic heart disease, neurodegenerative disorders and hair loss. On the other hand, an excessive and deregulated angiogenic response is thought to contribute to cancer, and other diseases [2]. More precisely, the angiogenetic formation of blood vessels in tumors, is an interactive process between tumor, endothelial and stromal cells in order to create a network for oxygen and nutrients supply, necessary for tumor growth. One of the most popular assay tissues to study the angiogenesis phenomenon

Charalampos N. Doukas is with the University of the Aegean, Dep. of Information \& Communication Systems Engineering, Samos, Greece. (phone: +30-2106010198, email: doukas@ieee.org)

Ilias Maglogiannis is with the University of the Aegean, Dep. of Information \& Communication Systems Engineering, Samos, Greece (phone: +30-22730-82239, email: imaglo@aegean.gr)

Aristotle Chatziioannou is with the Institute of Biological Research and Biotechnology, National Hellenic Research Foundation, Greece.

Andreas Papapetropoulos is with the Lab of Molecular Pharmacology, School of Pharmacy, University of Patras, Greece. is the developing chick embryo and its chorioallantoic membrane (CAM). CAM is a highly vascular structure lining the inner surface of the egg shell. The study of the formation of its vessels, at different stages of development, is efficiently indicative for the phenomenon ([7]-[12]). The aim of this study was to test and develop an automated image analysis method that would give an unbiased quantification of the micro-vessel density. The developed tool processes digital images of in vivo chick embryo and its CAM. Using advanced image processing techniques, the proposed tool exploits vessel characteristics, such us vessel length, density, vessel branching points and textural information to assess the level of angiogenesis.. The presented method has been validated by comparing automated results to manual expert assessment over a series of digital chick embryo images. The results indicate the high accuracy and reproducibility of the tool, which has been thus extensively used for tumor growth detection at different stages of embryonic development. The rest of the paper is organized as follows; Section 2 presents related work regarding CAM development and automated angiogenesis quantification. Section 3 describes the CAM angiogenesis procedure and Section 4 presents the proposed quantification methodology. Evaluation results are presented while, Section 6 concludes the paper.

\section{RELATED WORK}

CAM is one of the most widely used models to study angiogenesis. Attempts to quantify angiogenesis using biochemical measurements have focused on [14C] - proline and hemoglobin determination. Type IV colllagen, is abundantly expressed in the basement membrane, a structure that develops below and supports the vascular endothelium. Like all types of collagen, type IV collagen has high proline content. This method is used by a few investigators with some success [3]. Other laboratories use hemoglobin content as a marker for angiogenesis [4]. Most investigators prefer to quantify the angiogenic response in the CAM by manual scoring or by morphometric analysis of CAM pictures using appropriate software, such as Scion Image [5], or Image-Pro Plus [6]. The latter tools provide image processing techniques, which allow measurements of vascular length after being applied manually on the examined angiographic images. Additional work in literature ([9]-[12]) presents computer-aided methods for angiogenesis quantification 
which involve manual processing of the images for calculating vessel density, vessel length and branching points. To our knowledge there is no automated software for angiogenesis quantification currently available. In this context, the presented method provides an automated angiogenesis assessment based on vessel length, branching points and texture quantification. Additional features allow the calculation of vessel thickness and the automated process of numerous experimental images.

\section{EXPERIMENTAL SETUP AND IMAGE ACQUISITION}

This section describes how CAM and corresponding digital images are acquired for the experiment. Fertilised White Leghorn chicken eggs were placed in an incubator and kept under constant humidity at $37 \mathrm{oC}$. On day 4, a square window was opened in the shell and then sealed with adhesive tape. In experiments designed to monitor the development of vascular networks at different stages of development, tissues were collected on day $9,10,11$ or 12 . In a separate series of experiments we evaluated the effects of dexamethasone, a known angiogenesis inhibitor. For these experiments, an O-ring $(1 \mathrm{~cm} 2)$ was placed on the surface of the CAM on day 9 and dexamethasone (8nmol/egg) was placed inside this restricted area. After 48 hr, CAMs were fixed in Carson's solution (saline-buffered formalin) and color images of the collected tissues were obtained through a Canon Powershot A620 digital camera (7.1 MPixels) using a Carl Zeiss W10X/20 stereoscope (6.3$25 \mathrm{~mm}$ object field, up to $32 \mathrm{x}$ zoom). Obtained images were processed by the proposed image analysis tool presented in the next section that automates the assessment of the angiogenic procedure.

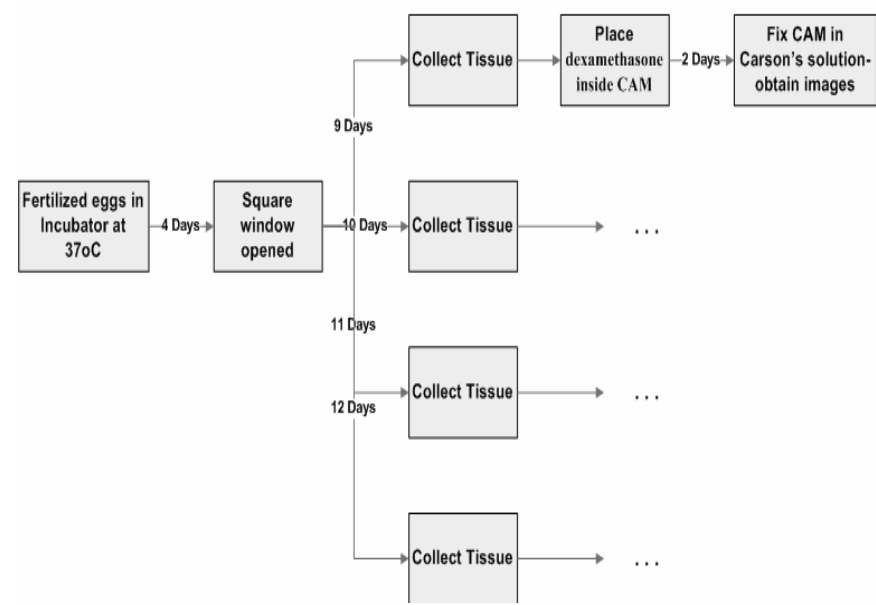

Figure 1. Experimental setup and image acquisition procedure

An illustration of the aforementioned experimental procedure is provided by Figure 1 .

\section{AUTOMATED ASSESSMENT OF ANGIOGENESIS IN STEREOSCOPIC IMAGES}

The automated processing and angiogenesis quantification of the stereoscopic images is performed in three basic phases (see Figure 2); during phase $\mathrm{A}$ the angiogenic image is captured and converted to grayscale. Phase B performs adaptive thresholding and noise removal. During adaptive thresholding, a user predefined size of pixel neighborhood is processed and the mean value $M$ is calculated. Then for every pixel value $p$, if $p<M-\mathrm{C}$, where $\mathrm{C}$ is a user defined constant, the $p$ is set to the background value. Otherwise, the foreground value is assumed. Noise removal follows using a Median Filter adapted from [13]. Additional noise removal techniques found in literature include conservative smoothing [14], Gaussian filtering [15], Mean filtering [16] and others [17]. Median filtering has been selected due its simplicity and low complexity, resulting in faster image processing, and efficient performance on the applied sample images. The image output of phase B is illustrated in Figure 3(b). Depending on the image initial quality (lighting conditions, vessel density, tissue quality), different values of the neighborhood size and the constant $\mathrm{C}$ can be selected for optimizing the output image quality of phase B. During our experiments the value of $\mathrm{C}$ that optimizes the thresholding procedure was $\mathrm{C}=15$ with a neighborhood size of $5 \times 5$ pixels. Comparing Figure 3(a) and Figure 3(b), it can be obvious that issues like bad lighting effects and tissue margins on the image corners are efficiently addressed by the adaptive thresholding procedure. Phase $\mathrm{C}$ skeletonizes the image and produces the final image output (Figure 3(c)) that contains vessels with thickness of one pixel. Finally, the developed tool calculates automatically the total vessel length, the vessel branching points, the vessel density over the whole image area and the vessel texture, assessing this way the angiogenesis phenomenon.

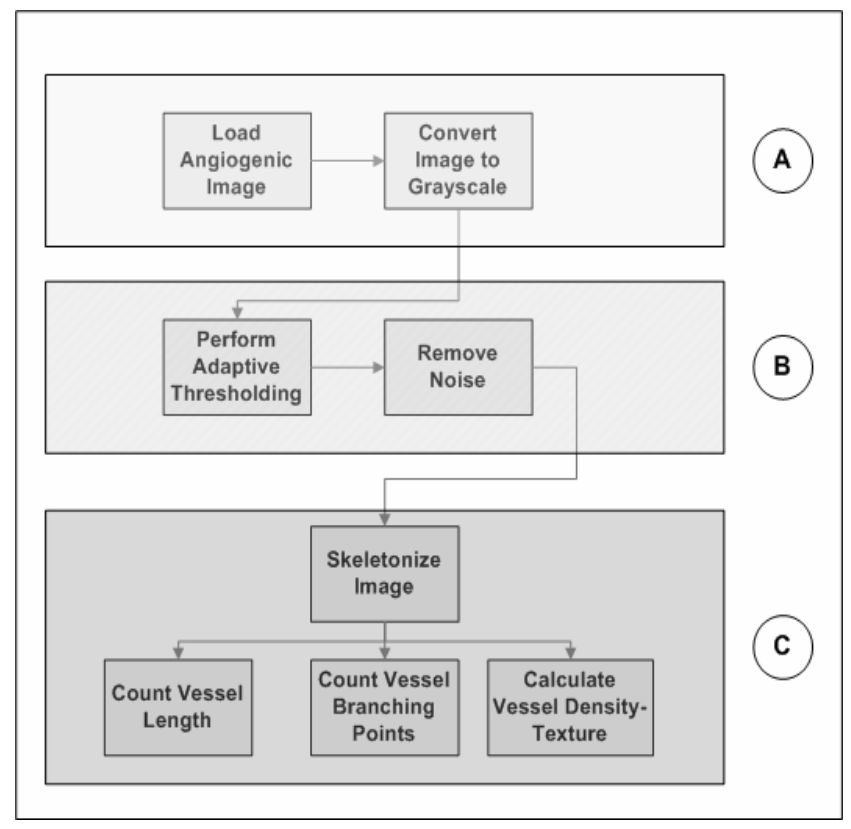


Figure 2. Block Diagram of the automated angiogenesis assessment

The aforementioned quantitative results are saved in XML files that can be directive saved into a database for processing. In addition, the tool offers automatic process of numerous image files defined by user, and basic image manipulation functions, like zooming, Region of Interest (ROI) selection and manual vessel thickness calculation.
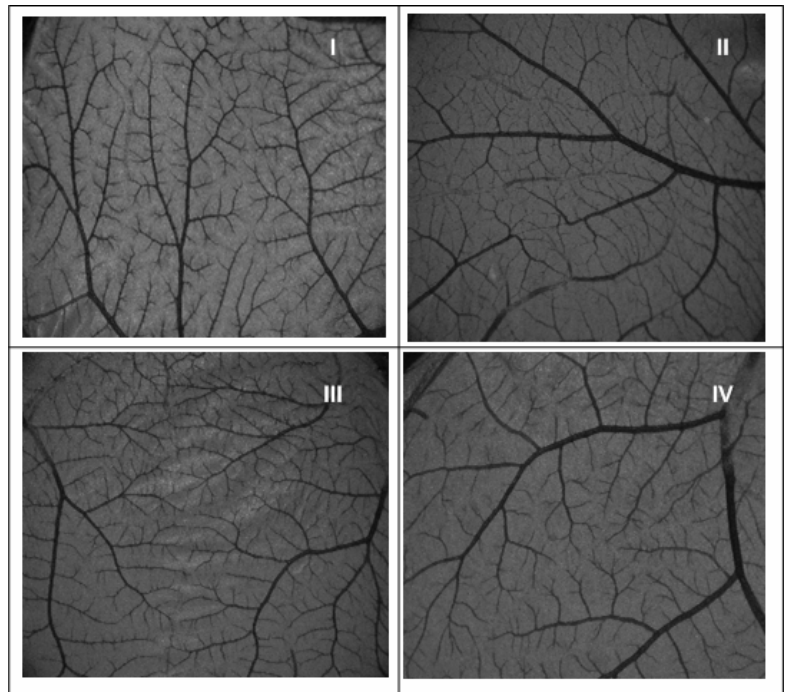

(a)

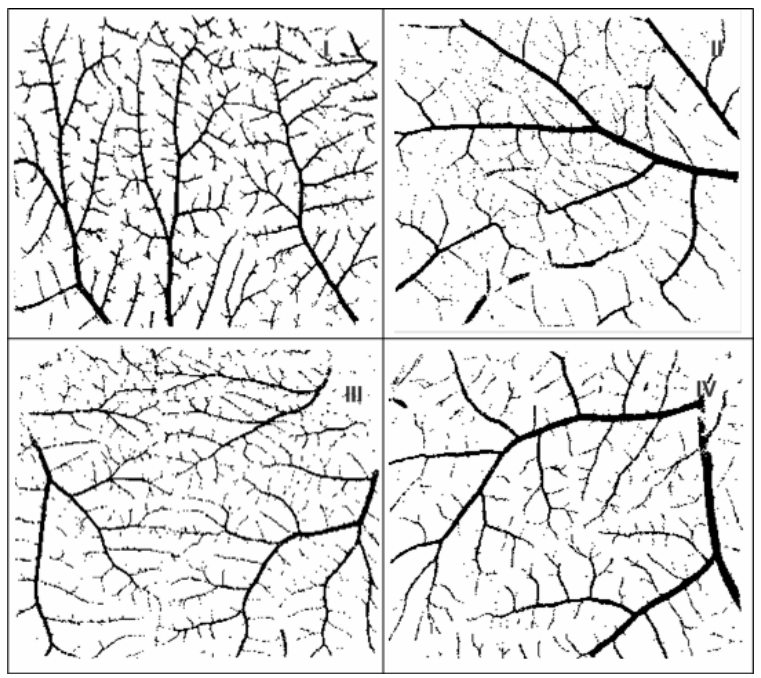

(b)

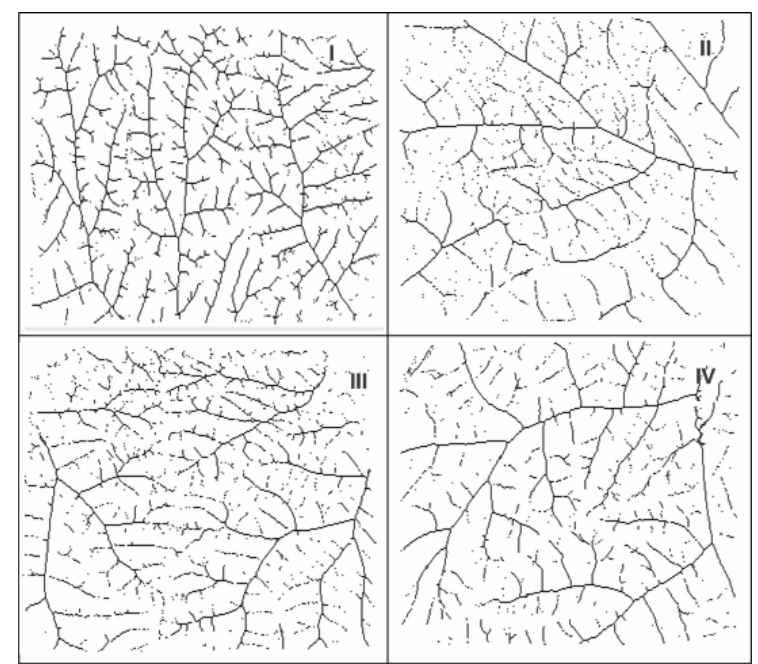

(c)

Figure 3. (a) Original angiogenic CAM images after greyscale conversion, (b) images produced after adaptive thresholding and noise removal, (c) skeletonized images

The corresponding results (i.e. vessel length, vessel density, branching points and image texture in terms of contrast, correlation and entropy) for the images included in Figure 3 are presented in Table I.

TABLE I

EXPERIMENTAL ANGIOGENESIS QUANTIFICATION RESULTS

\begin{tabular}{lllll}
\hline \hline \begin{tabular}{l} 
Vessel Quantification \\
\multicolumn{1}{c}{ Metric }
\end{tabular} & \multicolumn{4}{c}{ Value } \\
\hline $\begin{array}{l}\text { Image No. } \\
\text { Vessel Length } \\
\text { (pixels) }\end{array}$ & I & II & III & IV \\
Vessel density (\%) & 8.6 & 11.1 & 10.4 & 7.3 \\
Vessel Branching & 439 & 485 & 557 & 303 \\
points & & & & \\
Image Texture & & & & \\
Features & & & & \\
Contrast & 0.122 & 0.165 & 0.140 & 0.103 \\
Correlation & 4.028 & 3.025 & 3.542 & 4.565 \\
Entropy & 0.780 & 0.930 & 0.833 & 0.736 \\
\hline \hline
\end{tabular}

\section{EVALUATION RESULTS AND DISCUSSION}

The presented automated angiogenesis quantification method has been validated against manual counts of vessel branching points over a series (25) of angiogenic CAM images acquired with the procedure described in Section 3. The corresponding results are illustrated in Figure 4, which presents the normalized number of vessel branching points calculated or manually counted in each image divided by the mean value of branches in the whole image dataset. 


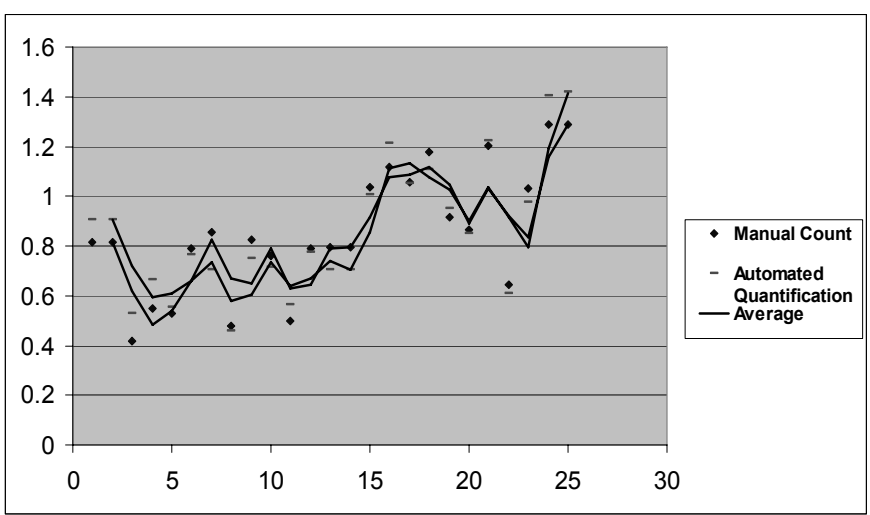

Figure 4. Automated Quantification validation results against manual counts over 25 CAM angiogenic images. Y axis represents the normalized number of vessel branches calculated or manually counted for each image, divided by the mean value of branching points in all images.

The Mean Average Error for the automated quantification of the experimental images against the manual count of branching points was 5\% with an average standard deviation of $7 \%$. The results indicate the high accuracy of the presented method in automated quantification of the vessel branching points. The latter conclusion can also avouch the accuracy of the additional calculated features (i.e. the vessel length, density and texture). The tool has been thus extensively used by biologists for quantification of the angiogenesis phenomenon and tumor growth detection and inhibition.

\section{CONCLUSION}

Automated quantification of angiogenesis levels is quite important in the objective assessment of drugs, chemical substances or anti-angiogenic procedures in general. The current methodology used by several medical labs is based on either on the visual count or in the use of commercially available image analysis SW (i.e. Scion Image, Image-Pro Plus, etc.). This methodology introduces large variation, caused by inter- or intra-operator sensitivity regarding the visual counts, or different setup of parameters and various processing steps regarding the existing image analysis SW. Quite important is also the standardization of image acquisition procedure which ensures the reproducibility of the captured images.

In this study, we aim at the implementation of a fully automated, objective, computer-based image analysis tool for the determination of angiogenesis level in digital stereoscopic images. At this stage we demonstrated that the implemented tool can calculate correctly several features, related to quantification of angiogenesis, such as vessel length, density and branching points. The produced results were highly correlated with the average human visual counts, conducted by experts from the Institute of Biological Research and Biotechnology in National Hellenic Research Foundation and in the Lab of Molecular Pharmacology, School of Pharmacy, University of Patras in Greece. Our future work involves the introduction of advanced classifiers in the proposed tool (Statistical, Neural Networks, Support
Vector Machines, etc) and the exploitation of various image textural features for achieving further quantification and automation in the assessment of angiogenesis level.

\section{REFERENCES}

[1] Jain, R., "Molecular regulation of vessel maturation", Nature Med, vol. 9, pp. 685-693, 2003.

[2] Ferrara, N., and Kerbel, R., "Angiogenesis as a therapeutic target", Nature vol. 438, pp. 967-974, December 2005.

[3] Maragoudakis ME, Panoutsacopoulou M, Sarmonika M., "Rate of basement membrane biosynthesis as an index to angiogenesis", Tissue Cell. vol. 20, no. 4, pp. 531-539, 1988.

[4] Chen J, Somanath PR, Razorenova O, Chen WS, Hay N, Bornstein P, Byzova TV. "Akt1 regulates pathological angiogenesis, vascular maturation and permeability in vivo", Nat Med. vol. 11, pp. 1188-1196, 2005.

[5] H. Loutrari, M. Hatziapostolou, V. Skouridou, E. Papadimitriou., C. Roussos, F.N. Kolisis and A. Papapetropoulos, "Perillyl alcohol inhibits angiogenesis in vitro and in vivo", J. Pharmacol. Exp. Therap. no.311, pp. 568-575, Nov. 2004.

[6] R. J. Blatt, A. N. Clark, J. Courtney, C. Tully, A. Tucker, “Automated quantitative analysis of angiogenesis in the rat aorta model using Image-Pro Plus 4.1", Computer Methods and Programs in Biomedicine, vol. 75, pp. 75-79, 2004.

[7] B. Weyn, W. A. Tjalma, P. Vermeylen, A. van Daele, E. van Marck, W. Jacob, "Determination of Tumor Prognosis Based on Angiogenesisrelated vascular Patterns Measured By Fractal and Synthetic Structure Analysis", Clinical Oncology, vol. 16, pp. 307-316, 2004.

[8] L. Sanz, M. Pascual, A. Munoz, M. Gonzalez, C. Salvador, L. Vallina, "Development of a Computer-Assisted High Throughput Screening Platform for Anti-angiogenic Testing", Microvascular Research, vol. 63, pp. 335-339, 2002.

[9] T. Krecicki, D. Dus, J. Kozlak, W. Trnawski, M. Jelen, M. Krekicka, T. Szkudlarek, "Quantitative evaluation of angiogenesis in laryngeal cancer by digital image measurement of the vessel density", Auris Nasus Larynx, vol. 29, pp. 271-276, 2002.

[10]R. Wild, S. Ramakrishnan, J. Sedgewick, W. Griffoen, "Quantitative Assesment of Angiogenesis and Tumor Vessel Architecture by Computer-Assisted Digital Image Analysis: Effects of VEGF-Toxin Conjugate on Tumor Microvessel Density", Microvascular Research, vol. 59, pp. 368-376, 2000.

[11]W. F. Seifert, A.A. Verhofstad, T. Wobes, W. Lange, P. Rijken, A. Kogel, T. Hendriks, "Quantitation of Angiogenesis in Healing Anastomoses of the Rat Colon", Eperimental and Molecular Pathology, vol.. 64, pp. 31-40, 1997.

[12] G. Nikiforidis, D. Papazafiropulos, D. Siablis, D. Karnabatidis, O. Hatjikondi, J. Dimopoulos, "Quantitative Assessment of angiogenesis in the chick embryo and its choriallantoic membrane by computerized analysis of angiographic images", European Journal of Radiology, vol.29, pp. 168-179, 1999.

[13]Loupas, T. McDicken, W.N. Allan, P., "An adaptive weighted median filter for speckle suppression inmedical ultrasonic images", IEEE Transactions on Circuits and Systems, vol. 36, pp. 129-135, Jan. 1989.

[14]Conservative smoothing description found at $\mathrm{http}: / /$ www.cee.hw.ac.uk/hipr/html/csmooth.html

[15]Deng, G. Cahill, L.W., "An adaptive Gaussian filter for noise reduction and edge detection", In Proc. of Nuclear Science Symposium and Medical Imaging Conference, pp. 1615-1619, 1993.

[16] Taguchi, A., Murata, Y., "The median and mean hybrid filters", In Proc. of IEEE International Symposium on Circuits and Systems, pp. 93-96, June 1991.

[17]Jamil, N., Abu Bakar, Z., Tengku Mohd Tengku Sembok, "A comparison of noise removal techniques in songket motif images", In Proc. of International Conference on Computer Graphics, Imaging and Visualization, pp. 139-143, July 2004. 